\title{
Convergence in per-capita GDP across European regions: a reappraisal
}

\author{
Valentina Meliciani ${ }^{1}$ and Franco Peracchi ${ }^{2}$ \\ ${ }^{1}$ Faculty of Economics, University of Teramo, I-00133, Rome, Italy + 1-801-626-7423 \\ (e-mail: jaap.wieling@xendo.nl)
}

Final version received: September 2003/ Final version received: July 2004

\begin{abstract}
This paper studies convergence in per-capita GDP across European regions over the period 1980-2000. We use median unbiased estimators of the rate of convergence to the steady-state growth path, while allowing for unrestricted patterns of heterogeneity and spatial correlation across regions. By permitting the model parameters to be completely different across regions, not only we avoid imposing strong a priori assumptions but we are also able to analyze the spatial patterns in the estimated coefficients. Our results differ from those found using conventional estimators. The main differences are: i) the mean rate of convergence is much lower; ii) for most regions this rate is zero; iii) the number of regions for which we reject equality in trend growth rates is substantially lower. We also find significant evidence of correlation of growth rates across neighbor regions and across regions belonging to the same country.
\end{abstract}

Key words: Regional convergence, median unbiased estimation, heterogeneous panel models

JEL classification: C23, O40, O52, R11

\section{Introduction}

This paper studies convergence in per-capita GDP across European regions over the period 1980-2000. The evidence currently available on regional convergence in Europe is mostly based on either cross-sectional "Barro regressions" or fixed-effects estimates. The results obtained vary considerably depending on the regions included, the sample period and the estimation method.

Using cross-sectional "Barro regressions", Barro and Sala-i-Martin (1991) found that regions within the European Union (EU) experienced convergent

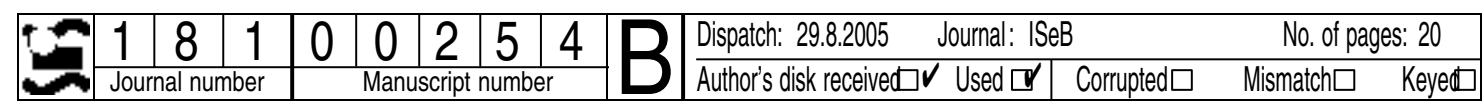


growth in per-capita GDP over the period 1950-1985 at an annual rate of about $2 \%$. Their analysis, however, is confined to the richest European countries. Extending the analysis to 1990 and including the Spanish regions, Sala-i-Martin (1996) still finds significant convergence (although at the lower rate of $1.5 \%$ ) in a regression that contains country dummies. Armstrong (1995) enlarges the sample to Greece, Ireland, Luxembourg and Portugal, and finds that the rate of convergence between 1970 and 1990 has been only some $1 \%$ per year. He concludes that rates of convergence, in particular within country convergence, fell from their peak in the 1960s. Neven and Gouyette (1995) also find big differences in the patterns of convergence across subperiods and across subsets of regions.

The fixed-effects approach, originally used by Islam (1995) to measure convergence across countries, has been applied to study regional convergence, among others, by Canova and Marcet (1995) for the European regions and by de la Fuente (1996) for the Spanish regions. All these studies obtain much higher convergence rates than those found in cross-country regressions. The convergence process has a different interpretation, however, for it is convergence to country- or region-specific steady-states. Moreover, the high estimated convergence rates are difficult to reconcile with the neoclassical growth theory, for they imply very low (and sometimes negative) capital shares. Canova and Marcet (1995), using a Bayesian estimator which permits the estimation of different convergence rates to different steady-states for each region, find evidence supporting lack of convergence in income levels but some convergence in growth rates. De la Fuente (1998) finds that explicitly allowing for short-term noise reduces the estimated rate of convergence to values which are roughly consistent with an extended neoclassical model.

Both cross-sectional "Barro regressions" and fixed-effects estimates place strong a priori restrictions on the model parameters. The former impose complete regional homogeneity in the parameters of the process that describes the evolution of per-capita GDP, while the latter allow for unobserved heterogeneity but confine differences across regions to the intercept of the model.

An alternative time-series approach to convergence has been developed by Bernard and Durlauf $(1995,1996)$. According to this approach a group of countries converge in output when the long-term forecasts of output for all countries are equal at a fixed time $t$, while countries have common trends in output if the long-term forecasts of output are proportional at a fixed time $t$. These definitions have natural testable counterparts in the cointegration literature. In fact, convergence requires countries' outputs to be cointegrated with cointegrating vector $[1,-1]$, while the existence of common trends only requires the output series to be cointegrated with cointegrating vector $[1,-\alpha]$. This approach does not impose the constraints imposed by cross-country and fixed effects approaches. However, it requires long time-series and does not allow estimating the different parameters of the process that drives the evolution of per-capita GDP, such as the convergence rate and the trend growth rate. ${ }^{1}$

\footnotetext{
${ }^{1}$ For completeness, another approach to study convergence in per-capita GDP is to focus on the evolution of its cross-sectional distribution. Using this methodology, Quah (1996) finds that while disparities have decreased between European countries, they have increased across regions within countries.
} 
Unlike previous studies at the regional level, this paper estimates separate processes for each region using the heterogeneous panel approach proposed by Lee, Pesaran and Smith (1997) for studying convergence in a panel of countries over the period 1960-1989. By permitting the model parameters to be completely different across regions, not only we avoid imposing strong a priori assumptions but we are also able to analyze the spatial patterns in the estimated coefficients. We also try to address some problems of this estimation method that have been recognized but not addressed by Lee, Pesaran and Smith.

First of all, conventional estimators of the autoregressive coefficient, which capture the rate of convergence to the steady-state growth path, are severely downward biased in short time series. Further, this bias translates into invalid inference about the other model parameters. To deal with these problems, we use median unbiased estimators of the autoregressive parameter, as proposed by Andrews (1993), and construct confidence sets for the other parameters based on these median unbiased estimates.

Second, most panel studies of convergence ignore cross-sectional correlation in the regression errors. This is particularly implausible when studying convergence across regions, as contemporaneous shocks are likely to affect simultaneously different regions within the same country, and possibly also across countries. In this paper, we take into account the possibility of crosssectional correlation by treating regional relationships as a system of seemingly unrelated regression equations.

The remainder of this paper is organized as follows. Section 2 presents the basic statistical model and its economic interpretation. Section 3 discusses the issues that arise when trying to allow for complete regional heterogeneity in the model parameters, and describes how they are addressed. Section 4 presents the data used in the empirical analysis. Section 5 reports the results obtained. Finally, Section 6 offers some concluding remarks.

\section{The statistical model}

The basic statistical model in the empirical literature on convergence is the deterministic linear trend model with AR(1) errors

$$
\begin{aligned}
Y_{i t} & =c_{i}+g_{i} t+U_{i t} \\
U_{i t} & =\lambda_{i} U_{i, t-1}+\epsilon_{i t},
\end{aligned}
$$

where $Y_{i t}$ is the $\log$ of per-capita GDP of region $i$ at time $t, \lambda \in(-1,1]$, and $\epsilon_{i t}$ is an innovation with constant variance $\sigma_{i}^{2}$. Notice that innovations may be contemporaneously correlated across regions. The parameters $c_{i}$ and $g_{i}$ respectively measure the mean initial level and the mean growth rate of per-capita GDP in region $i$, whereas the autoregressive parameter $\lambda_{i}$ measures the degree of persistence of the shocks to log per-capita GDP in region $i$. The parameter $v_{i}=-\ln \lambda_{i}$, defined for $\lambda_{i}>0$, measures the speed of convergence of per-capita GDP in region $i$ to its long-run growth path $c_{i}+g_{i} t$, and will be referred to as the "rate of convergence".

The growth equations that are often estimated in cross-sectional studies (the so-called "Barro regressions") can be obtained from (1) by imposing equality across regions in all parameters $\left(c_{i}, g_{i}, \lambda_{i}\right)$, while the growth equations 
estimated in the context of fixed-effects models can be obtained by imposing homogeneity in the parameters $g_{i}$ and $\lambda_{i}$, leaving the $c_{i}$ unrestricted.

If $\lambda_{i}=1$, the intercept $c_{i}$ is not identifiable and model (1) reduces to $Y_{i t}-Y_{i, t-1}=g_{i}+\epsilon_{i t}$, namely a random walk with drift $g_{i}$. In this case, given two regions $i$ and $j$ with $\lambda_{i}=\lambda_{j}=1$, it makes sense to talk about convergence only if the processes for log per-capita GDP in the two regions are cointegrated. Irrespective of whether it makes sense to talk about convergence, one may well have $g_{i}=g_{j}$, that is, the same average growth rate of per-capita GDP in two regions.

Equation (1) may arise as the reduced form of several growth models. Most empirical studies focus on the neoclassical Solow's growth model (Solow 1956) with no uncertainty, an aggregate Cobb-Douglas production function, initial level of technology $A_{0}$, capital share $\alpha$, depreciation rate of the capital stock $\delta$, savings rate $s$, growth rate of labor input $m$ and growth rate of technology $g$. Except for $A_{0}$, all the model parameters are assumed to be time invariant, although they may differ across regions (henceforth, we drop the subscript $i$ whenever this causes no ambiguity). In this model, the dynamic equation for log per-capita GDP is given by

$$
Y_{t}=(1-\lambda)(c+g t)+\lambda g+\lambda Y_{t-1},
$$

where $\lambda=e^{-v}, v=(m+g+\delta)(1-\alpha)$ is the the rate of convergence, and the parameter $c$ depends on all the model parameters through the relationship

$$
c=\left[\ln A_{0}+\frac{\alpha}{1-\alpha} \ln \left(\frac{s}{m+g+\delta}\right)\right] .
$$

Adding an innovation $\epsilon_{t}$ to the deterministic relationship (2) and rearranging terms gives a representation which is equivalent to (1).

More recently, Lee, Pesaran and Smith (1997) have developed a stochastic version of the neoclassical growth model where both technology and employment follow AR(1) processes with a linear trend and possibly a unit root. In this model, countries might experience different growth rates even if they have access to the same technology. Equation (1) may be obtained as a reduced form of this model under somewhat stringent assumptions on the correlation between the employment and the technology shock, and the order of magnitude of their autocorrelation coefficients. In this case, the coefficient on the lagged dependent variable also depends on the amount of serial correlation in the technology shocks. In particular, a unit root in output may arise either because of constant marginal productivity of capital $(\alpha=1)$ or a unit root in technology.

\section{Methodology}

Unlike previous studies at the regional level, this paper estimates equation (1) separately for each region, thus allowing for unrestricted parameter heterogeneity and arbitrary correlation in the innovations across regions. This enables us to investigate the extent of convergence and the patterns of spatial correlation across European regions without imposing a priori strong homogeneity restrictions. 
Estimation and inference about the parameters of model (1) is rather tricky. In carrying out the strategy of estimating the model parameters separately for each region, we need to address three issues: (i) the downward bias in the traditional estimates of the autoregressive parameter $\lambda$, (ii) the quality of the inference about the intercept $c$ and the slope $g$ of the time trend, and (iii) the likely correlation of the innovations across regions. As we argue below, the way in which the autoregressive parameter is estimated turns out to be crucial, for it affects inference (point estimation and hypothesis testing) about other parameters, even in the absence of any correlation of the innovations across regions.

\subsection{Estimation of $\lambda$}

The most common estimators of $\lambda$ are the coefficient on $Y_{t-1}$ in an OLS regression of $Y_{t}$ on a constant, a linear trend and $Y_{t-1}$, and various estimators obtained from the residuals $\hat{U}_{t}$ in an OLS regression of $Y_{t}$ on a constant and a linear trend, such as $\hat{\lambda}=\sum_{t=7}^{T} \hat{U}_{t} \hat{U}_{t-1} / \sum_{t=3}^{T} \hat{U}_{t-1}^{2}$ (the unconditional LS estimator), $\bar{\lambda}=\sum_{t=2}^{T} \hat{U}_{t} \hat{U}_{t-1} / \sum_{t=2}^{t} \hat{U}_{t-1}^{2}$ (the conditional LS estimator) and the coefficient of sample correlation between $\hat{U}_{t}$ and $\hat{U}_{t-1}$. Notice that only the last estimator guarantees that the estimates of $\lambda$ will lie within the parameter space $(-1,1]$. Although consistent, all these estimators are known to be downward biased in finite samples, and the size of their bias increases with the absolute value of $\lambda$ and decreases with the sample size $T$. Not allowing for this bias represents one of the main flaws of existing studies on convergence.

Several ways of correcting conventional estimators of $\lambda$ for their bias have been proposed in the literature (see for example Quenouille 1956 and Orcutt $\&$ Winokur 1969). In this paper, we follow the procedure suggested by Andrews (1993), which corrects for median bias. We then use the resulting median unbiased estimates of $\lambda$ to carry out inference about the parameters of the time trend.

An estimator of $\lambda$ is said to be median unbiased if, for any $\lambda$, its sampling median is equal to $\lambda$. A median unbiased estimator has the "impartiality" property that the probability of overestimating and underestimating the true parameter $\lambda$ are the same.

Andrews (1993) presents a method for constructing median unbiased estimators of $\lambda$ in Gaussian AR(1) models. His method may be used to biascorrect any estimator of $\lambda$ with a continuous and strictly increasing distribution function and a sampling median that is continuous and strictly increasing in $\lambda$ for $-1<\lambda \leq 1$. Notice that the parameter space includes the case of a unit root process and therefore allows for a smooth transition between the trend stationary case $(|\lambda|<1)$ and the unit root case $(\lambda=1){ }^{2}$ Given an estimator $\hat{\lambda}$ with median function $\zeta(\cdot)$, a median unbiased estimator of $\lambda$ is

\footnotetext{
2 The method has two limitations. First, it only applies to AR(1) processes. An approximately median unbiased estimator for the AR( $p$ ) model has been proposed by Andrews and Chen (1994). Second, it requires knowledge of the shape of the distribution of the innovations. Numerical results presented by Andrews (1993) show that procedures based on the normality assumption are robust to a variety of nonnormal distributions.
} 


$$
\tilde{\lambda}= \begin{cases}1, & \text { if } \hat{\lambda}>\zeta(1), \\ \zeta^{-1}(\hat{\lambda}), & \text { if } \zeta(-1)<\hat{\lambda} \leq \zeta(1), \\ -1, & \text { otherwise, }\end{cases}
$$

where $\zeta^{-1}()$ is the inverse of $\zeta()$ and $\zeta(-1)=\lim _{\lambda \rightarrow-1} \zeta(\lambda)$. Notice that, by construction, $\tilde{\lambda}$ belongs to the interval $(-1,1]$. To see why $\tilde{\lambda}$ is median unbiased notice that, by definition, its median is equal to the median of $\zeta^{-1}(\hat{\lambda})$. If $\zeta^{-1}$ is continuous and strictly increasing on $(-1,1]$, it then follows that the median of $\tilde{\lambda}$ is equal to $\zeta^{-1}($ med $\hat{\lambda})=\zeta^{-1}(\zeta(\lambda))=\lambda$. Implementation of this method typically relies on numerical evaluation of the median $\zeta(\lambda)$ of $\hat{\lambda}$ on a fine grid of $\lambda$ values, and interpolation to obtain the median function $\zeta(\cdot)$ and its inverse $\zeta^{-1}(\cdot)$.

Lee, Pesaran and Smith (1997) point out that the main drawback of median unbiased estimators of $\lambda$ is their large sampling variance relative to conventional estimators. In the remainder of this section we investigate whether this larger sampling variance is more than offset by the smaller bias.

We report summary statistics based on a set of Monte Carlo experiments for a sample of 21 observations from model (1) with Gaussian innovations. Each experiment consists of 10,000 replications and corresponds to a different value of $\lambda$ in the range $[-0.98,1.00]$, at intervals of width .02 . The same set of pseudo-random numbers is used in each experiment. The conventional estimator of $\lambda$ is again the coefficient on $Y_{t-1}$ in an OLS regression of $Y_{t}$ on a constant, a linear trend and $Y_{t-1}$.

We exploit two important properties of the model, namely the fact that when $|\lambda|<1$ and the initial value $Y_{0}$ is random, the sampling distribution of the conventional estimator depends only on $\lambda$ and the sample size $T$, while when $\lambda=1$ it does not depend on the initial value $Y_{0}$ (see Andrews 1993 for a proof). Thus, we set $c=g=0$. For $|\lambda|<1$, we randomly draw the innovations from the $\mathscr{N}(0,1)$ distribution and the starting value $Y_{0}$ from the $\mathscr{N}\left(0,\left(1-\lambda^{2}\right)^{-1}\right)$ distribution, whereas for $\lambda=1$ we set $Y_{0}=0$.

Figure 1 compares the median bias, the mean bias, the standard error (SE), and the root mean square error (RMSE) of the sampling distribution of the two estimators of $\lambda$. The figure shows that the downward bias of the conventional estimator is very large. For example, its mean bias is equal to -.214 for $\lambda=.60,-.277$ for $\lambda=.80,-.325$ for $\lambda=.90$ and -.363 for $\lambda=.96 .^{3}$ Using the conventional estimator therefore leads to severely underestimate the autoregressive coefficient and to severely overestimate the rate of convergence. Notice that the sampling median of the conventional estimator is strictly increasing in $\lambda$, which is what is required for constructing median unbiased estimators. ${ }^{4}$

The small-sample bias of the conventional estimator represents a problem for any empirical study of convergence based on short time series. For example, the sample of OECD countries used by Lee, Pesaran and Smith (1997) consists of 29 annual observations. In this case, when $\lambda=1$, the sampling median of the conventional estimator of $\lambda$ can be shown to be

\footnotetext{
${ }^{3}$ Detailed tables are available from the authors upon request.

${ }^{4}$ We have no formal proof that the quantiles of the conventional estimator are strictly increasing in $\lambda$, although numerical calculations for various sample sizes show this to be the case (Andrews 1993).
} 


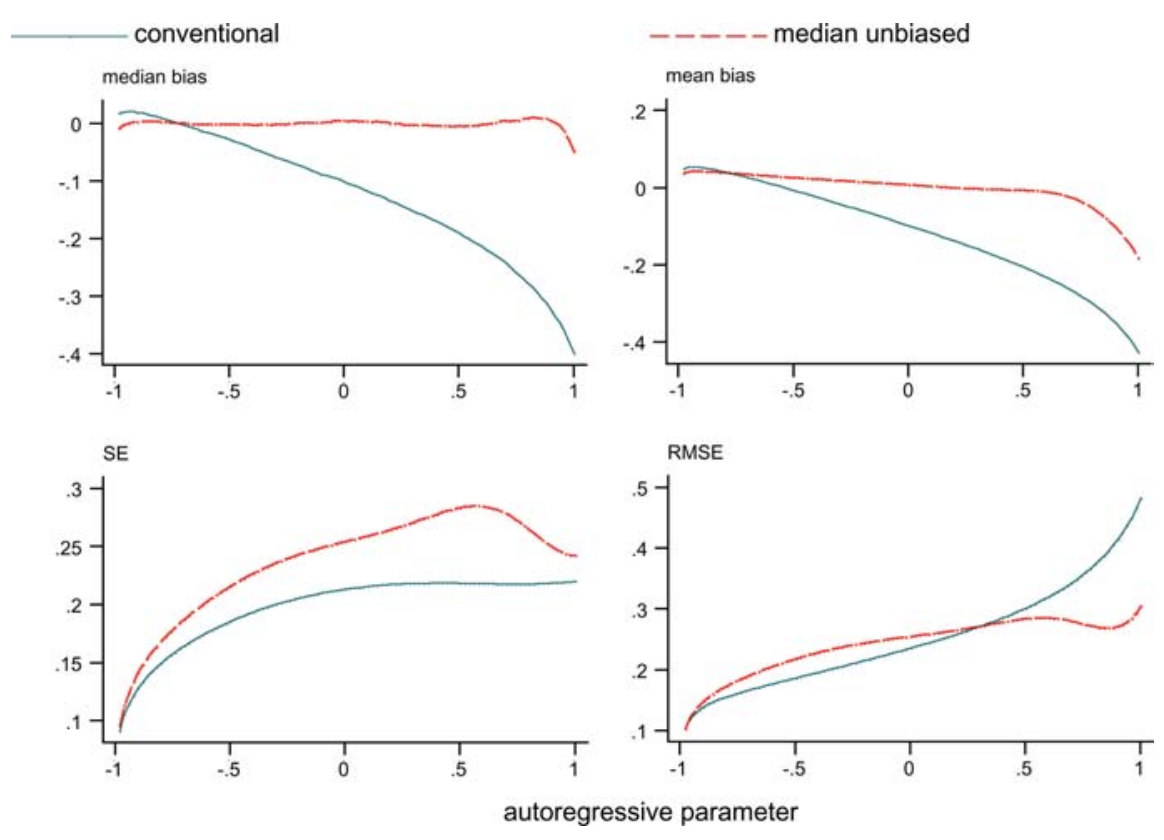

Fig. 1. Median bias, mean bias, standard error (SE) and root mean square error (RMSE) of conventional and median unbiased estimators of $\lambda$

equal to $.678 .^{5}$ Considering that the cross-country median of their estimates of $\lambda$ is .789 (see their Table 1, p. 370), for more than half of the countries the median unbiased estimator of $\lambda$ would be equal to 1 , implying no convergence. This may explain why their estimates show fast convergence but are nevertheless unable to reject the null hypothesis of a unit root in output.

Although the median unbiased estimator always has larger standard error and smaller mean bias than the conventional estimator, the difference in the variability of the two estimators does not increase with $\lambda$, while the difference in the bias does. In fact, while the bias and the standard error of the conventional estimator are strictly increasing in $\lambda$, the standard error of the median unbiased estimator actually decreases for $\lambda>.58$. It turns out that, for values of the autoregressive parameter above .32, the larger variance of the median unbiased estimator relative to the conventional one is more than offset by its smaller bias. Thus, for values of $\lambda$ corresponding to those typically found in convergence studies, the median unbiased estimator has smaller root mean square error than the conventional one. ${ }^{6}$ The efficiency of the median unbiased estimator relative to the conventional one depends of course on the sample size, and is typically reversed in large samples.

\footnotetext{
${ }^{5}$ Tables are available from the authors upon request.

${ }^{6}$ The same experiment carried out for other conventional estimators of $\lambda$ confirms these results. Moreover, all conventional procedures provide very similar results in terms of mean bias, median bias, standard errors and RMSE.
} 


\subsection{Inference about the time trend}

Several estimators are available for the parameters $(c, g)$ in model (1). The OLS estimator in a regression of $Y_{t}$ on a constant and the linear trend is unbiased but inefficient. Its inefficiency vanishes in large samples, however, because the columns $(1,1, \ldots, 1)$ and $(0,1, \ldots, T-1)$ of the design matrix are close to being linear combinations of two characteristic vectors of the covariance matrix of an $\mathrm{AR}(1)$ process. $^{7}$

When $|\lambda|<1$ is known, the best linear unbiased estimator of $(c, g)$ is the GLS estimator, obtained by applying OLS to the data transformed using the feasible GLS (Prais-Winsten) transformation. When $\lambda=1$, the parameter $c$ is not identifiable and the GLS estimator of $g$ is just the sample average of the differences $Y_{t}-Y_{t-1}$. When $\lambda$ is unknown, a feasible GLS estimator, asymptotically equivalent to GLS, is easily obtained by "plugging-in" a consistent estimate of $\lambda$.

The approximate GLS estimator proposed by Cochrane and Orcutt (1949) is instead quite inefficient in finite samples, even when $\lambda$ is known, especially for $\lambda$ close to unity. The source of the inefficiency is the omission of the first observation. The problems with the Cochrane-Orcutt estimator worsen considerably when $\lambda$ is unknown.

The finite-sample properties of all these estimators have been investigated by Park and Mitchell (1980) and Canjels and Watson (1997). The two studies show that, when $\lambda$ is estimated in a conventional way, the Cochrane-Orcutt estimator is always less efficient than OLS, while feasible GLS estimators based on the Prais-Winsten transformation (either two-step or fully iterated) offer efficiency gains over OLS that range from modest to substantial depending on the value of $\lambda$ and the sample size. For large values of $\lambda$, feasible GLS estimators appear to have a slight edge in small samples over the exact maximum likelihood procedure based on the normality assumption. Because of these results, we henceforth focus on feasible GLS estimators of (1).

When a feasible GLS procedure is used, the way in which $\lambda$ is estimated is crucial. First, the feasible GLS transformation breaks down when the estimates of $\lambda$ are greater than one in absolute value. Second, biased estimation of $\lambda$ may reduce the efficiency gain from using a feasible GLS estimator. Third, and most importantly, they may imply higher probabilities of Type I error than nominal.

In fact, the Monte Carlo evidence in Park and Mitchell (1980) reveals large discrepancies between the actual and the nominal level of Wald tests on the trend coefficient when $\lambda$ is positive and conventional estimates of $\lambda$ are used. To see the source of the problem, notice that, under model (1), the sampling variance of the exact GLS estimator $\hat{g}$ is equal to $\operatorname{Var}(\hat{g})=q_{1} \sigma^{2} /\left(q_{1} q_{3}-q_{2}^{2}\right)$, where $q_{1}, q_{2}$ and $q_{3}$ are the following functions of $\lambda$ and the sample size $T$

\footnotetext{
${ }^{7}$ Chipman (1979) showed that the greatest lower bound for the efficiency of the OLS estimator of $g$ over the interval $0 \leq \lambda<1$ is equal to .7534, approached as $T \rightarrow \infty$ and $\lambda \rightarrow 1$.
} 


$$
\begin{aligned}
& q_{1}=1-\lambda^{2}+\sum_{t=1}^{T-1}(1-\lambda)^{2}=(1-\lambda)[T(1-\lambda)+2 \lambda], \\
& q_{2}=(1-\lambda) \sum_{t=1}^{T-1}[t-\lambda(t-1)]=(T-1)(1-\lambda)\left[\frac{T}{2}(1-\lambda)+\lambda\right], \\
& q_{3}=\sum_{t=1}^{T-1}[t-\lambda(t-1)]^{2}=T(T-1)(1-\lambda)\left[\frac{2 T-1}{6}(1-\lambda)+\lambda\right]+(T-1) \lambda^{2} .
\end{aligned}
$$

This sampling variance increases monotonically with $\lambda$ for $T$ fixed. Estimating $\operatorname{Var}(\hat{g})$ by "plugging-in" a downward biased estimator of $\lambda$ leads to underestimate the sampling variance of $\hat{g}$ and therefore to incorrectly reject a null hypothesis about $g$ with a probability that is larger than the nominal size of the test.

Figure 2 reports the results of a set of Monte Carlo experiment that analyzes the actual level of a $t$ test of significance of the linear trend in model (1) estimated by feasible GLS with alternative estimates of $\lambda$. The setup of the experiments is exactly the same as in Section 3.1. The figure compares the actual frequencies of Type I error for nominal 5\%-level two-sided tests based on conventional and median unbiased estimators of $\lambda$. Except for values of $\lambda$ close to -1 , the actual level of the test is always higher than the nominal and the discrepancy between the actual and the nominal level increases with $\lambda$. The frequency of Type I error is much larger, however, when the conventional estimator of $\lambda$ is used. For example, when $\lambda=.60$ the test based on the conventional estimator rejects in $17.3 \%$ of the cases, when $\lambda=.80$ it rejects in

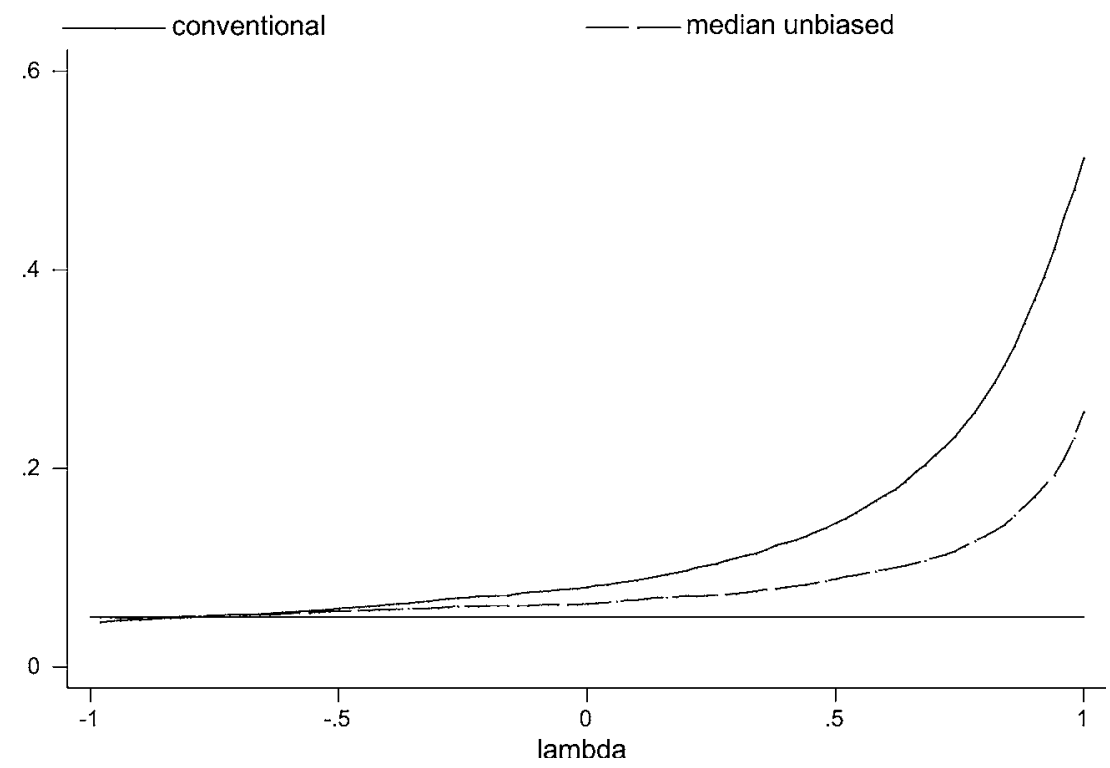

Fig. 2. Monte Carlo frequency of Type I error for a nominal 5\%-level two-sided $t$ test of significance of the linear trend in model (1) estimated by exact GLS using conventional and median unbiased estimators of $\lambda$. 
$27.1 \%$ of the cases, when $\lambda=.90$ it rejects in $37.0 \%$ of the cases, and when $\lambda=.96$ it rejects in $45.3 \%$ of the cases. On the other hand, when $\lambda=.60$ the test based on the median unbiased estimator rejects in $9.8 \%$ of the cases, when $\lambda=.80$ it rejects in $13.1 \%$ of the cases, when $\lambda=.90$ it rejects in $17.1 \%$ of the cases, and when $\lambda=.96$ it rejects in $20.9 \%$ of the cases.

The use of median unbiased estimators of $\lambda$ therefore goes a long way towards reducing the discrepancy between the actual and the nominal level of a test, thus providing a simple and viable alternative to the use of generalized bounds tests, as proposed by Dufour (1990), or asymptotically conservative tests, as proposed by Canjels and Watson (1997).

Our final concern is the possible correlation of the innovations across regions. It is hard to justify the assumption that innovations in two different regions are uncorrelated. In fact, correlation is likely to be present either between regions in the same country (because of common country-specific shocks) or between adjacent regions in different countries (because of trade and spillover effects). Thus, when testing for equality across regions of the parameters of the time trend one should deal with the fact that the crosssectional correlation in the innovations may lead to invalid inference if not properly taken into account.

Lee, Pesaran and Smith (1997) try to remove the contemporaneous correlation by transforming the data in deviations from the country-specific mean. In fact, their procedure is only justified when countries (regions) have the same value of the autoregressive parameter and when the common shocks have the same impact across all countries (regions).

In this paper, we follow an alternative route. First we remove the autocorrelation by using the median unbiased estimates of the region-specific autocorrelation coefficient to transform the observations via the exact GLS transformation. We then test for equality of the time trend coefficients between pairs of regions by estimating a seemingly unrelated regression equations (SURE) model on the transformed data in order to take into account the possible correlation in the innovations. ${ }^{8}$

\section{The data}

Our data come from the REGIO database of Eurostat and are categorized according to the Nomenclature of Statistical Territorial Units (NUTS). Although this categorization consists of three levels (NUTS1, NUTS2 and NUTS3, with NUTS1 corresponding to the coarsest level and NUTS3 to the finest), none of them can be considered as fully satisfactory (Boldrin \& Canova 2000). For this reason, we rely instead on the alternative categorization proposed by Paci (1997) and Rodríguez-Pose (1998).

The selected categorization follows two criteria: (i) comparable levels of self-government in countries with a sufficient degree of administrative decentralization (Germany, Belgium, Spain, Italy, France, and partially Portugal and the United Kingdom) and, (ii) comparable size in terms of territory or population for the remaining countries (Denmark, Greece, Ire-

\footnotetext{
${ }^{8}$ Phillips and Sul (2003) show that, in the case of short time series with high degrees of cross sectional dependence, the SURE median unbiased estimator has MSE performance that is 5 times better than that of the OLS estimator and twice as good as that of the SURE estimator.
} 
land, Luxembourg, and the Netherlands). It selects regional units corresponding to the following administrative levels: Régions for Belgium, Régions for France, Länder for Germany, Groups of Development regions for Greece, Regioni for Italy, Landsleden for the Netherlands, Regioes autonomas for Portugal, Communidades autónomas for Spain, and Standard regions for the UK. The resulting categorization coincides with NUTS 1 for Belgium, Germany, Greece, Netherlands and the UK, and with NUTS 2 for France, Italy, Portugal and Spain. Denmark, Ireland and Luxembourg are each treated as a single region.

A further complication is the fact that, in late 1998, the NUTS has been revised to incorporate changes in the administrative structure of the various countries. There were minor revisions for Finland, Germany and Sweden, but major revisions for the UK. To ensure comparability over time, whenever possible we reclassify the data for 1995-2000 according to the old NUTS. For Germany, we exclude the Eastern Ländern and some other regions for which there is no correspondence between the old and the new NUTS. Moreover we exclude Brussels and three UK regions (North, North-West and South-East) for which data were not comparable across the two classifications. The resulting sample consists of 95 regions followed for each year from 1980 to 2000 (see Table 1$)^{9}$.

GDP data have been converted to a common scale using purchasing power parities (PPPs) rather than exchange rates, since the latter do not take into account differences in purchasing power across countries. Growth rates are computed using per-capita GDP in 1995 PPPs and prices. Due to lack of regional price indices, data have been deflated using the national consumer price index.

\section{Empirical results}

We estimate model (1) separately for each of the 95 European regions using both conventional and median unbiased estimators of $\lambda$. After presenting the results obtained under different estimation procedures (Section 5.1), we discuss the evidence on spatial correlation (Section 5.2) and parameter heterogeneity (Section 5.3).

\subsection{Parameter estimates}

Table 2 reports summaries of the distribution of the estimates of the model parameters $c, g$ and $\lambda$ across regions. Within the neoclassical growth model, $c$ is the steady-state level of per-capita GDP in the absence of technical change, whereas $g$ is the rate of technical change. We also report summaries of the rate of convergence parameter $v=-\ln \lambda$.

The table shows the results obtained when the model is estimated under different assumptions on parameter heterogeneity. The cross-sectional estimates assume a common rate of convergence and a common steady-state level of per-capita GDP. Notice that only the parameter $\lambda$ can be estimated in this case. Fixed-effects estimates allow for region-specific values of $c$ but assume a

\footnotetext{
${ }^{9}$ The location of the regions in a geographical map is reported in Meliciani and Peracchi (2004).
} 
Table 1. List of the European regions considered

\begin{tabular}{|c|c|c|c|c|c|}
\hline be2 & Vlaams Gewest & es62 & Murcia & it4 & Emilia-Romagna \\
\hline be3 & Région Wallonne & es63 & Ceuta y Melilla & it51 & Toscana \\
\hline $\mathrm{dk}$ & Denmark & es7 & Canarias & it52 & Umbria \\
\hline del & Baden-Württemberg & fr 1 & Ile de France & it53 & Marche \\
\hline de2 & Bayern & fr21 & Champagne-Ardenne & it6 & Lazio \\
\hline de 5 & Bremen & fr22 & Picardie & it71 & Abruzzo \\
\hline $\operatorname{de} 6$ & Hamburg & fr23 & Haute-Normandie & it72 & Molise \\
\hline de7 & Hessen & fr24 & Centre & it8 & Campania \\
\hline de9 & Niedersachsen & fr 25 & Basse-Normandie & it91 & Puglia \\
\hline dea & Nordrhein-Westfalen & fr26 & Bourgogne & it92 & Basilicata \\
\hline deb & Rheinland-Pfalz & fr3 & Nord-Pas-de-Calais & it93 & Calabria \\
\hline dec & Saarland & fr41 & Lorraine & ita & Sicilia \\
\hline def & Schleswig-Holstein & $\mathrm{fr} 42$ & Alsace & itb & Sardegna \\
\hline gr1 & Voreia Ellada & fr43 & Franche-Comté & lu & Luxembourg \\
\hline gr2 & Kentriki Ellada & fr51 & Pays de la Loire & $\mathrm{nll}$ & Noord-Nederland \\
\hline gr3 & Attiki & fr52 & Bretagne & $\mathrm{nl} 2$ & Oost-Nederland \\
\hline gr4 & Nisia Aigaiou, Kriti & fr53 & Poitou-Charentes & $\mathrm{nl} 3$ & West-Nederland \\
\hline es11 & Galicia & fr61 & Aquitaine & $\mathrm{nl} 4$ & Zuid-Nederland \\
\hline es12 & Principado de Asturias & fr62 & Midi-Pyrenees & pt11 & Norte \\
\hline es13 & Cantabria & fr63 & Limousin & pt12 & Centro \\
\hline es 21 & Pais Vasco & fr71 & Rhóne-Alpes & pt13 & $\begin{array}{l}\text { Lisboa e Vale } \\
\text { do Tejo }\end{array}$ \\
\hline es 22 & Comunidad de Navarra & fr72 & Auvergne & pt14 & Alentejo \\
\hline es 23 & La Rioja & fr81 & Languedoc-Roussillon & pt15 & Algarve \\
\hline es24 & Aragón & fr82 & Prov-Alpes-Cóte Azur & uk2 & $\begin{array}{l}\text { Yorkshire and } \\
\text { Humberside }\end{array}$ \\
\hline es3 & Comunidad de Madrid & ie & Ireland & uk3 & East Midlands \\
\hline es41 & Castilla y León & it11 & Piemonte & uk4 & East Anglia \\
\hline es42 & Castilla-la Mancha & it12 & Valle d'Aosta & uk6 & South West \\
\hline es43 & Extremadura & it13 & Liguria & uk7 & West Midlands \\
\hline es51 & Cataluña & it2 & Lombardia & uk9 & Wales \\
\hline es52 & Comunidad Valenciana & it31 & Trentino-Alto Adige & uka & Scotland \\
\hline es53 & Baleares & it32 & Veneto & $\mathrm{ukb}$ & Northern Ireland \\
\hline es61 & Andalucia & it33 & Friuli-Venezia Giulia & & \\
\hline
\end{tabular}

common value of $g$ and $\lambda$. Finally, heterogeneous panel models allow all three parameters to be region-specific. In this case, we report both the conventional and the median unbiased estimates of the autoregressive parameter. For the other parameters ( $c$ and $g$ ) we report the GLS estimates based on these alternative estimates of $\lambda$.

The rate of convergence ranges from a value of .016 for the cross-sectional case, to .13 for the fixed-effect estimates, to a mean value of .53 for the heterogeneous panel estimates based on the conventional estimates of $\lambda$. Our fixed-effects and heterogeneous panel estimates of the rate of convergence are much larger than those obtained by Lee, Pesaran and Smith (1997) at the country level for a sample of 22 OECD countries. In fact, they obtain a value of .95 for $\lambda$ (implying a value of .05 for the rate of convergence) when only allowing for heterogeneity in $c$, and a value of .76 for $\lambda$ (implying a value of .27 for the rate of convergence) when allowing for complete heterogeneity (see their tables 1 and 4). Our higher estimates could depend, in part, on the fact that our data refer to regions rather than countries. They may also be a consequence of the fact that the downward bias in the autoregressive coeffi- 
Table 2. Summary of parameter estimates $(v=-\ln \lambda)$

\begin{tabular}{|c|c|c|c|c|}
\hline Cross-section regression & $c$ & $g$ & $\lambda^{\lambda} .984$ & $\begin{array}{l}v \\
.016\end{array}$ \\
\hline \multicolumn{5}{|l|}{ Fixed-effects estimates } \\
\hline Mean & 9.226 & .027 & .880 & .127 \\
\hline Standard deviation & .259 & & & \\
\hline Minimum & 8.687 & & & \\
\hline Lower quartile & 9.075 & & & \\
\hline Median & 9.216 & & & \\
\hline Upper quartile & 9.365 & & & \\
\hline Maximum & 9.966 & & & \\
\hline \multicolumn{5}{|c|}{ Heterogeneous panel using $\hat{\lambda}$ (conventional) } \\
\hline Mean & 9.353 & .022 & .623 & .527 \\
\hline Standard deviation & .314 & .009 & .191 & .450 \\
\hline Minimum & 8.543 & -.002 & -.076 & .021 \\
\hline Lower quartile & 9.150 & .015 & .533 & .294 \\
\hline Median & 9.374 & .020 & .663 & .409 \\
\hline Upper quartile & 9.575 & .025 & .745 & .625 \\
\hline Maximum & 10.170 & .054 & .980 & 3.578 \\
\hline \multicolumn{5}{|c|}{ Heterogeneous panel using $\tilde{\lambda}$ (median unbiased) } \\
\hline Mean & 9.261 & .021 & .879 & .185 \\
\hline Standard deviation & .284 & .009 & .201 & .442 \\
\hline Minimum & 8.556 & .000 & .034 & .000 \\
\hline Lower quartile & 9.088 & .015 & .826 & .000 \\
\hline Median & 9.358 & .020 & 1.000 & .000 \\
\hline Upper quartile & 9.462 & .024 & 1.000 & .191 \\
\hline Maximum & 9.618 & .054 & 1.000 & 3.368 \\
\hline
\end{tabular}

cients (and therefore the upward bias in the rate of convergence) is larger for shorter time series (see Andrews, 1993). Since our time series consists of 21 observations while the Lee, Pesaran and Smith time series consists of 29 observations, the upward bias in the rate of convergence should be larger for our estimates. In fact, the table shows that the mean rate of convergence falls from .53 to about .18 if we use median unbiased rather than conventional estimators of $\lambda$. Further, for more than half of the regions the median unbiased estimator of $\lambda$ is equal to one, implying no convergence. Also note that the trend growth rate is higher for fixed effects estimates (0.027) than for heterogeneous panel estimates $(0.022$ and 0.021 for the results based respectively on the conventional and on the median unbiased estimates of $\lambda$ ).

Figure 3 is a map of Europe with the value of the estimates of the trend growth rate. Higher values of the estimates correspond to darker colors in the map. Looking at the map, there is evidence of both spatial and national effects in the distribution of the trend growth rate. The highest growth rates are found in all the Portuguese regions, several Spanish regions, Ireland, Luxembourg, the Greek Islands and two Italian regions (Trentino-Alto Adige, Veneto).

The Spanish regions with the highest trend growth rate are Ceuta-y-Melilla, Canarias, Comunidad de Madrid, Extremadura, Cataluña, Aragón, Comunidad de Navarra, Balears, Murcia, Comunidad Valenciana, Castilla-la Mancha, Pais Vasco and Castilla-y-León. This group includes regions with per-capita incomes both above and below the national average. The UK regions appear to have intermediate trend growth rates, while the French regions 


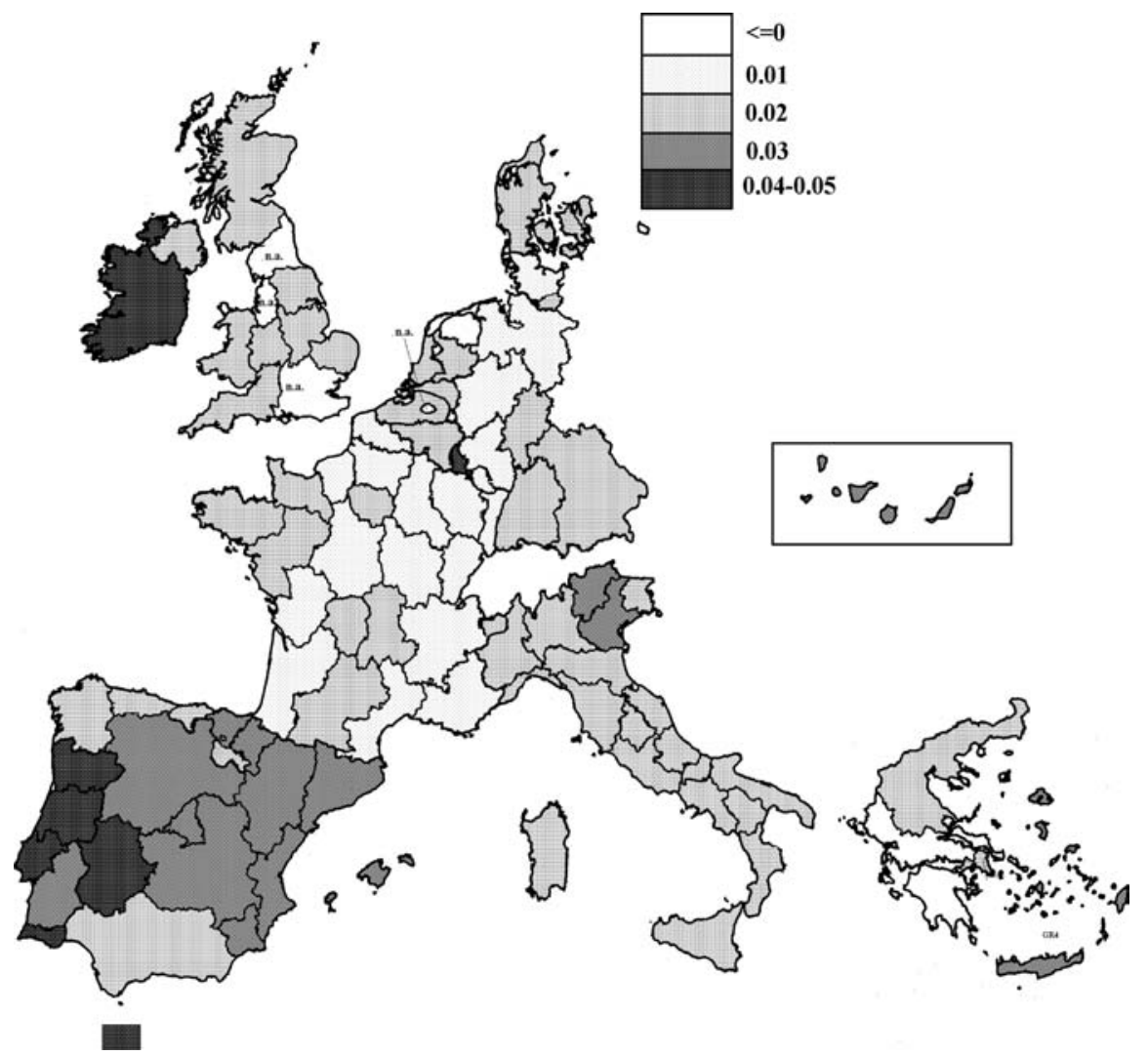

Fig. 3. Estimates of the trend growth rate

tend to have below average growth rates. In general laggard countries, with the exception of Greece, appear to experience above-average mean growth rates. However, the same tendency does not appear to emerge across regions within the same country. ${ }^{10}$

\subsection{Spatial correlation}

The visual impression of spatial correlation in the trend growth rate may be investigated more formally. A popular indicator of spatial correlation is the Moran coefficient, defined as

$$
I=\frac{S^{-1} \sum_{i=1, i \neq j}^{n} \sum_{j=1}^{n} w_{i j}\left(x_{i}-\bar{x}\right)\left(x_{j}-\bar{x}\right)}{n^{-1} \sum_{i=1}^{n}\left(x_{i}-\bar{x}\right)^{2}},
$$

\footnotetext{
${ }^{10}$ The results for the trend growth rate estimated using conventional estimates of $\lambda$ do not differ much from the ones reported in the map (based on median unbiased estimates of $\lambda$ ).
} 

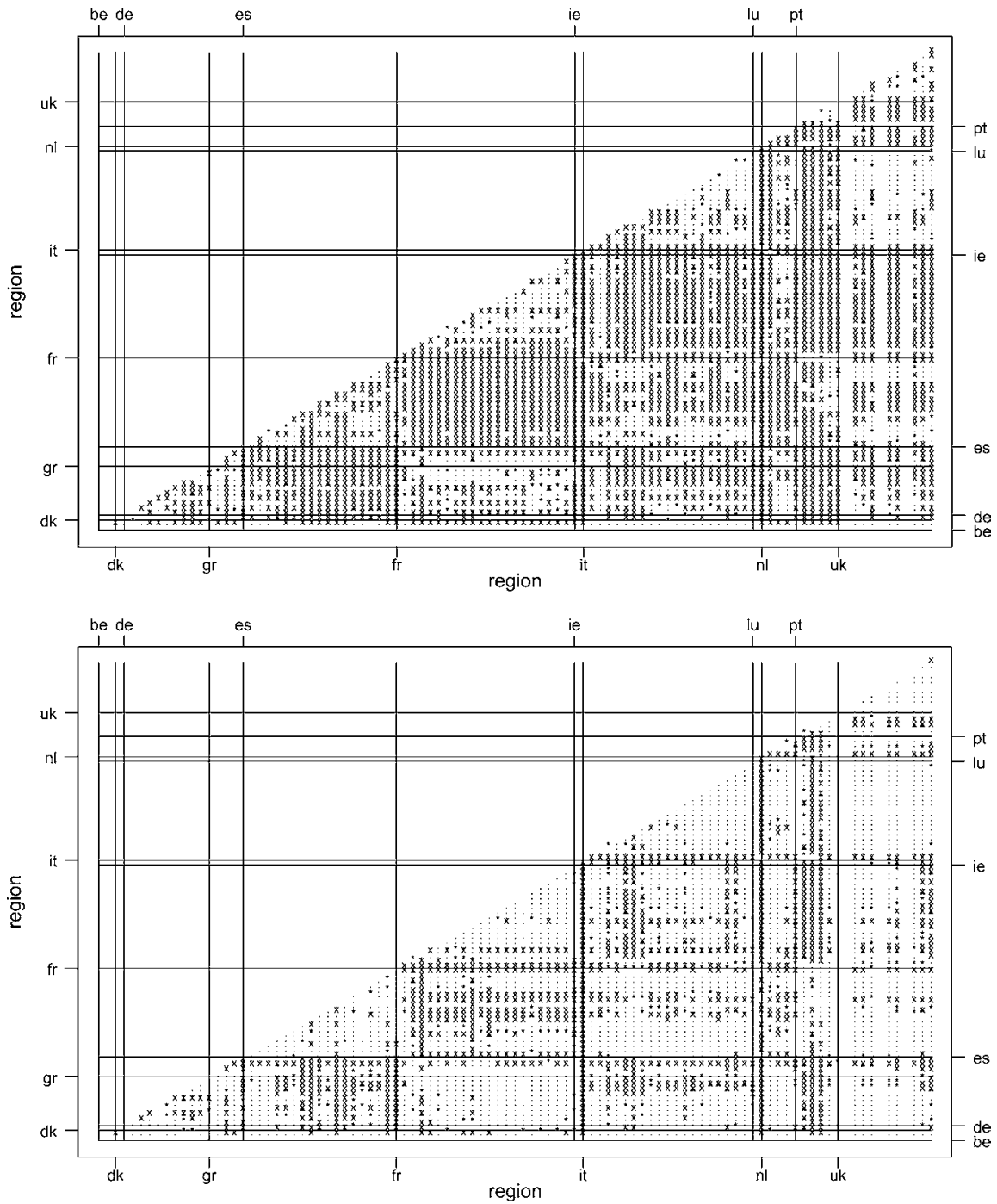

Fig. 4. Tests of equality of the trend growth rates between pairs of regions based on SURE estimates that allow for contemporaneous correlation in the innovations across regions. The top and bottom panels correspond, respectively, to the conventional and median unbiased estimates of $\lambda$. The symbol " $\mathrm{x}$ " indicates rejection at the 5\% level, "*" indicates rejection at the $10 \%$ level, "." indicates no rejection. The critical values are based on the $t$ distribution with 19 degrees of freedom

where $x_{i}$ is the value of the variable under consideration in region $i, \bar{x}$ denotes the average value of the variable across all regions, $n$ is the total number of regions, $w_{i j}$ denotes the generic element of an $n \times n$ matrix of weights, called the contiguity matrix, and $S=\sum_{i=1, i \neq j}^{n} \sum_{j=1}^{n} w_{i j}$. The Moran coefficient takes the classic form of any autocorrelation coefficient: the numerator measures 
the covariance among the $x_{i}$ and the denominator measures the variance. ${ }^{11}$ Because the Moran coefficient is asymptotically normally distributed under some regularity conditions (see Cliff \& Ord 1973, Chapter 1), inference on the significance of spatial correlation may be based on the standardized values of $I^{12}$

The specification of the contiguity matrix is crucial for the Moran coefficient. We consider three different specifications. The first assigns a weight of one when two regions share the same border and a weight of zero otherwise. This matrix we be referred to as the "neighbor matrix". To investigate to what extent spatial correlation might be due to country effects, we construct a "foreign neighbor matrix", by considering only border regions and by assigning a weight of one when two regions belonging to two different countries share the same border and a weight of zero otherwise. We also consider a "country matrix" that assigns a weight of one when two regions belong to the same country and a weight of zero otherwise. These matrices are used to compute the amount of neighbor, foreign neighbor and country correlation in the trend growth rate.

The value of the Moran coefficient changes little across estimation methods ${ }^{13}$. The trend growth rate is highly correlated for regions belonging to the same country (the correlation coefficient is 0.60 for both conventional and median unbiased estimators). It is also highly correlated for neighboring regions, but correlation is lower across neighbors than across regions in the same country (for neighboring regions the correlation coefficient is 0.46 using conventional estimators and 0.44 using median unbiased estimators). Moreover, when we compute the Moran coefficient excluding regions belonging to the same country, the correlation is still positive but statically insignificant. This indicates the presence of important country effects in regional trend growth rates. In the neoclassical growth model, the trend growth rate $g$ represents the rate of growth of technology. Following this interpretation, it appears that, in spite of the further integration of European regions, the diffusion of technology remains faster within one country than across the borders.

Overall the results show that, while there is little evidence of convergence to each region's steady-state per-capita GDP, ${ }^{14}$ there is some evidence of catching-up since the estimates of the trend growth rate of most regions in some laggard countries (Spain and Portugal) are higher than the average. On the other hand, the fact that trend growth rates are similar for regions within the same country independently of their initial levels of per-capita GDP, is consistent with the lack of within-country convergence in levels found by many studies on regional growth in Europe (see e.g. Boldrin \& Canova 2000).

\footnotetext{
${ }^{11}$ Rather than imposing any a priori constraint on spatial correlation in the coefficients or the error term of the model, we prefer to allow for complete heterogeneity in the coefficients and arbitrary patterns of correlation in the residuals and to use the Moran coefficient as a descriptive tool that summarizes the spatial distribution of the estimated coefficients.

${ }^{12}$ For the form of the asymptotic mean and standard deviation of $I$, see Cliff and Ord 1973.

${ }^{13} \mathrm{We}$ concentrate on the trend growth rate because for more than half of the regions the estimates of the autoregressive parameter $\lambda$ are equal to one and the intercepts are not defined.

${ }^{14}$ This result can be interpreted as evidence against decreasing marginal productivity of capital within the Solow growth model, but is also consistent with a unit root in technology in the stochastic version of the model.
} 


\subsection{Testing for parameter heterogeneity}

As already discussed, using conventional estimates of the autoregressive coefficient could lead to reject the null hypothesis more frequently than the nominal size of the test. Here we compare the results obtained using GLS estimators based on alternative estimates of the autoregressive parameter $\lambda$. In either case, we compare the results obtained not taking and taking into account the contemporaneous cross-sectional correlation in the innovations.

The number of pairwise tests of equality of the trend slope $g$ is equal to $n(n-1) / 2=95(94) / 2=4,465$. For the intercept $c$, the number of pairwise tests of equality depends instead on the number of regions for which the estimated value of $\lambda$ is less than one in absolute value, as the parameter is only identified in this case. Since this number is rather small, we focus on tests of homogeneity in trend growth rates.

The amount of heterogeneity in the estimated trend growth rates is significantly reduced when the GLS transformation is carried out using the median unbiased estimates rather than the conventional one. This is true independently of whether or not we also allow for contemporaneous correlation in the innovations. Ignoring the contemporaneous correlation (GLS) and using conventional estimates of $\lambda$, equality in $g$ is rejected at the $5 \%$ level in $54.5 \%$ of the cases, and at the $10 \%$ level in $62.0 \%$ of the cases. Using median unbiased estimates of $\lambda$, equality in $g$ is instead rejected at the $5 \%$ level in only $18.5 \%$ of the cases and at the $10 \%$ level in only $25.4 \%$ of the cases. When taking into account the contemporaneous cross-section correlation in the innovations (SURE), rejection rates at the 5\% (10\%) level go up to $64.4 \%(70.7 \%)$ if conventional estimates of $\lambda$ are used, and to $25.9 \%$ $(34.5 \%)$ if median unbiased estimates are used.

Figure 4 reports in more detail the results of our pairwise tests of equality of the trend growth rate based on SURE estimates that allow for contemporaneous correlation in the innovations across regions. ${ }^{15}$ The top and bottom panels correspond, respectively, to the conventional and median unbiased estimates of $\lambda$. Each point of a panel represents a pair of regions. The symbol " $\mathrm{x}$ " indicates rejection of equality at the 5\% level, "*" indicates rejection at the $10 \%$ level, while "." indicates no rejection. The critical values of the tests are based on the $t$ distribution with 19 degrees of freedom. Data are ordered so that regions belonging to the same country are close to each other. Vertical and horizontal lines mark the shift from one country to the next. It is therefore possible to discern from the figure the presence of country effects in homogeneity (heterogeneity) of trend growth rates. Due to the symmetry of each matrix, we have drawn the results of the tests only for the part below the diagonal.

Again we can observe that there are important country effects. In particular, on the basis of conventional $t$-tests most of growth homogeneity is found across regions belonging to the same country. On the other hand the trend growth rate of most French regions appears to be significantly different from the trend growth rate of most Spanish and Portuguese regions and from Luxembourg and Ireland. On the basis of corrected $t$-tests growth heteroge-

${ }^{15}$ A similar figure obtained ignoring cross-sectional correlation in the innovations is reported in Meliciani and Peracchi (2004). 
neity occurs in few cases (mostly involving Ireland, Luxembourg and some Portuguese, Italian, Spanish and French regions).

Finally, we investigate the relevance of taking into account the cross-sectional autocorrelation in the disturbances using the Breusch and Pagan (1980) test statistic. Since we are carrying out pairwise comparisons, the test statistic is simply equal to $T \hat{R}_{i j}^{2}$, where $\hat{R}_{i j}^{2}$ is the sample correlation between the GLS residuals from the $i$ th and the $j$ th region. Cross-sectional correlation in the innovations is statistically significant at the 5\% level in $36.7 \%$ of the cases when using conventional estimates of $\lambda$, and in $33.5 \%$ of the cases when using median unbiased estimates of $\lambda$. The cases of no autocorrelation prevail in the UK, Portugal and Greece, suggesting that these countries have experienced shocks which are different from the rest of the EU. ${ }^{16}$ The large number of cases of significant autocorrelation across regions (also belonging to different countries) suggests the importance of taking into account the covariance in the innovations when testing for equality in the parameters.

\section{Conclusions}

This paper analyzes convergence in per-capita GDP across European regions using a very standard model (a deterministic linear trend model with AR(1) errors) but trying to overcome some of the problems arising with previous empirical studies that have ignored the regional heterogeneity in the model parameters and the short time series dimension of the available data.

Heterogeneity in the model parameters has been addressed using heterogeneous panel estimators instead of more restrictive "Barro regressions" or fixed-effects estimators, whereas the issues arising from the short time series dimension of the data have been addressed by using median unbiased estimators of the autoregressive parameter in the model. Our Monte Carlo simulations show that, for values of the autoregressive parameter commonly found in convergence studies, the larger sampling variability of median unbiased estimators relative to conventional estimators is more than compensated by the smaller bias, resulting in a sampling distribution that is more concentrated about the target parameter.

We find that, for more than half of the European regions considered, the value of the median unbiased estimator is equal to one, implying no convergence to a steady-state level of per-capita GDP. The mean rate of convergence across regions using median unbiased estimators is about .18, less than half the value found using conventional estimators. These results suggest that there are serious problems in estimating the rate of convergence from short time series without properly taking into account the downward bias in the conventional estimates of the autoregressive parameter.

Conventional $t$ tests on the parameters of the linear trend in the model would also lead to reject the null hypothesis of equality with a probability that is much larger than the nominal size of the test. Moreover, the discrepancy between the actual and the nominal size increases with the value of the autoregressive parameter. To address this problem we have carried out $t$

\footnotetext{
${ }^{16}$ For a visual inspection of the patterns of correlation in the innovations across regions see Meliciani and Peracchi (2004), Figure 9.
} 
tests on the parameters of the linear trend replacing the conventional estimates of $\lambda$ with median unbiased ones. To test hypotheses on the equality of the parameters across regions we have also taken into account the crosssectional dependence in the error term.

While tests based on conventional estimates of $\lambda$ reject growth homogeneity in a majority of cases, tests based on median unbiased estimates of $\lambda$ lead to the conclusion that regional trend growth rates differ in a minority of cases. Further, by allowing all parameters to differ across regions, this study also reveals strong spatial patterns of correlation in the trend growth rates. We find that, despite the increasing integration among European regions, trend growth rates are still highly correlated between regions belonging to the same country. If the trend growth rate captures the rate of growth of technology, as suggested by the neoclassical growth model, it appears that the diffusion of technology is still easier within one country than across the borders.

We thank Badi Baltagi, Michele Boldrin, David Levine, Hashem Pesaran and Melvyn Weeks for useful comments on an earlier draft of this paper. Financial support from CNR and MIUR is gratefully acknowledged.

\section{References}

Andrews DWK (1993) Exactly median unbiased estimation of first order autoregressive/unit root models. Econometrica 61: 139-165

Andrews DWK, Chen HY (1994) Approximately median unbiased estimation of autoregressive models. Journal of Business and Economic Statistics 12: 187-414

Armstrong HW (1995) Convergence among regions of the European Union 1950-1990. Papers in Regional Science 74: 143-152

Barro RJ, Sala-i-Martin X (1991) Convergence across states and regions. Brookings Papers on Economic Activity 137-158

Bernard AB, Durlauf SN (1995) Convergence in international output. Journal of Applied Econometrics 10: $97-108$

Bernard AB, Durlauf SN (1996) Interpreting tests of the convergence hypothesis. Journal of Econometrics 71: 161-173

Boldrin M, Canova F (2000) Inequality and convergence: reconsidering European regional policies. Economic Policy 32: 207-253

Breusch TV, Pagan AR (1980) The LM test and its applications to model specification in econometrics. Review of Economic Studies 47: 239-254

Canjels E, Watson MW (1997) Estimating deterministic trends in the presence of serially correlated errors. Review of Economics and Statistics 79: 184-200

Canova F, Marcet A (1995) The poor stay poor: Non-convergence across countries and regions. CEPR Discussion Paper n. 1265

Chipman JS (1979) Efficiency of least squares estimation of linear trend when residuals are autocorrelated. Econometrica 47: 115-128

Cliff AD, Ord JK (1973) Spatial Autocorrelation. Pion Limited, London

Cochrane D, Orcutt GH (1949) Application of least squares regression to relationships containing autocorrelated error terms. Journal of the American Statistical Association 43: 32-61

de la Fuente A (1996) On the sources of convergence: a close look at the Spanish regions. CEPR Discussion Paper n. 1543

de la Fuente A (1998) What kind of regional convergence? CEPR Discussion Paper n. 1924

Dufour JM (1990) Exact tests and confidence sets in linear regressions with autocorrelated errors. Econometrica 59: 475-494

Islam N (1995) Growth empirics: A panel data approach. Quarterly Journal of Economics 110: $1127-1170$ 
Lee K, Pesaran MH, Smith R (1997) Growth and convergence in a multi-country empirical stochastic Solow model. Journal of Applied Econometrics 12: 357-392

Meliciani V, Peracchi F (2004) Convergence in per-capita GDP across European regions: A reappraisal. CEIS Working Paper n. 204

Neven D, Gouyette C (1995) Regional convergence in the European Community. Journal of Common Market Studies 33: 47-65

Orcutt GH, Winokur HS (1969) First order autoregressions: Inference, estimation and prediction. Econometrica 37: 1-14

Paci R (1997) More similar and less equal: Economic growth in the European regions. Weltwirtschaftliches Archiv 133: 609-633

Park RE, Mitchell BM (1980) Estimating the autocorrelated error model with trended data. Journal of Econometrics 13: 185-201

Phillips PCB, Sul D (2002) Dynamic panel estimation and homogeneity testing under cross section dependence. Cowles Foundation Discussion paper n. 1362.

Quah D (1996) Regional convergence clusters across Europe. European Economic Review 40: 286-952

Quenouille MH (1956) Notes on bias in estimation. Biometrika 43: 353-360

Rodríguez-Pose A (1998) The Dynamics of Regional Growth in Europe. Social and Political Factors. Clarendon Press, Oxford

Sala-i-Martin X (1996) Regional cohesion: Evidence and theories of regional growth and convergence. European Economic Review 40: 1325-1352

Solow R (1956) A Contribution to the theory of economic growth. Quarterly Jounal of Economics 70: 65-94 